\title{
A Working Classification of Retraction for the Whole Tympanic Membrane
}

\author{
G. D. Barr \\ Department of Otolaryngology, Head and Neck Surgery, Monklands Hospital, Airdrie, UK \\ Email: gdbarr@ntlworld.com
}

Received May 10, 2013; revised June 11, 2013; accepted June 30, 2013

Copyright (C) 2013 G. D. Barr. This is an open access article distributed under the Creative Commons Attribution License, which permits unrestricted use, distribution, and reproduction in any medium, provided the original work is properly cited.

\begin{abstract}
Objective: To formulate a reliable classification of tympanic membrane retraction which is easy to use but capable of detecting small differences in retraction. Study Design: Prospective study. Methods: The classification was developed from observations dividing the drum into 3 areas: the pars tensa anterior to the malleus, type I, posterior to the malleus (subdivided into upper and lower) type II, and attic type III. The subclassification on paper is more complicated but using a database it is easy to use. The classification is automatically calculated by the database which can also be converted to numerical form. The classification also allows documentation of active disease in retractions. Photographs of seven retracted ear drums were incorporated into the database and ten otolaryngologists asked to classify them. Five were asked to reclassify the retractions in the same way after 3 months. Results: Intra class correlation was significantly high ( $>0.9)$ for pars tensa, attic, and for bony erosion. Cronbach’s alpha values were also high $(>0.9)$ in all groups. Retest values were evaluated with Wilcoxon's signed rank sum test establishing that there was no significant difference in results. Conclusion: The classification shows reliabiliy and validity allowing detection of small changes in tympanic membrane retraction especially affecting the pars tensa but allows classification of the whole tympanic membrane.
\end{abstract}

Keywords: Tympanic Retraction; Atelectasis; Otitis Media

\section{Introduction}

Several classifications of tympanic membrane retraction exist, more so for the pars tensa compared to the pars flaccida. The number of classifications itself suggests that there has not been general satisfaction with any one type. Certainly the traditional Sade classification demonstrates the progression of retraction but not specifically where in the pars tensa it is occurring and can therefore be regarded as more pathophysiological than anatomical [1]. When trying to analyse the effect of treatment on retractions, in particular elevation in children with middle ear effusions at the time of grommet insertion, it was found that the current classifications could not demonstrate small changes of improvement. In addition there can be uncertainty regarding the subgroups of the Sade and Tos classifications which has led to their reliability being questioned [2]. For instance a small localised retraction of the pars tensa could be Sade III or IV but also a diffuse collapse of the whole drum could also be Sade III or IV. Equally the existing classifications do not allow recording of small changes when disease progresses during follow up.
The objective of this study was to formulate a reliable classification of tympanic membrane retraction which is easy to use but capable of detecting small differences in retraction which would be of use in assessing disease progression or assessment before and after surgery.

\section{Materials and Methods}

The classification was formulated from observation of retractions over more than two decades. The division between II upper (upp) and II lower (low) is estimated just inferior to the oval window niche corresponding to the level between the ponticulum and subiculum (Figure 1).

The subgroups are further divided numerically in a progressive manner with the full classification being shown in Tables 1(a) and (b).

Concerning the retraction at the incus or stapes, for II upp 2 this means the drum is flush with the incus or head of stapes or a slight concavity where it is draped over the ossicle but not greater than the diameter of the long process of the incus or stapes head itself. For each part of the drum the classification progresses towards complete 


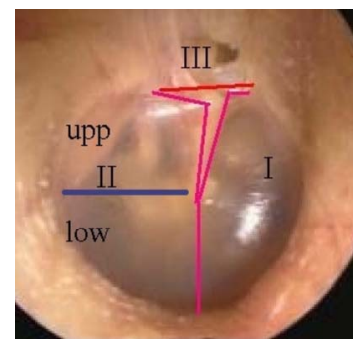

Figure 1. Image showing division of the tympanic membrane into Anterior (I), Posterior (II) with upper part of pars tensa (Upp) and lower (Low), and Attic (III).

Table 1. (a) Tympanic membrane retraction classification with subgroups; (b) Additional annotations to the classification.

(a)

\begin{tabular}{l} 
I [anterior drum] \\
\hline - $\quad 1$ Dimple/slight generalised retraction \\
- $\quad 2$ Deeper retraction not reaching promontory/medial wall \\
- $\quad 3$ Localised retraction to promontory/medial wall \\
- $\quad 4$ Complete retraction \\
II (upp) [posterior drum] \\
- 1 Slight retraction \\
- 2 Retraction reaching incus/stapes \\
- $\quad 3$ Retraction over incus/stapes (greater than diameter of stapes \\
- $\quad 4$ Retraction reaching footplate/facial nerve \\
II (low) [posterior drum] \\
- 1 Retraction not reaching promontory \\
- $\quad 2$ Reaching promontory/round window \\
- 3 More diffuse retraction to lower medial wall \\
III [attic] \\
- 1 Pit small retraction \\
- 2 Deeper retraction past the neck of malleus end visible \\
- 3 Deeper retraction past the neck of malleus end not visible \\
\hline
\end{tabular}

(b)

\section{Additional annotations}

- E Outer attic wall bone erosion present

Activity

- K: keratin; R: removable; $\mathrm{N}$ : not removable

- P: polyp; G: granulation

- D: discharge

- U: unclassifiable

Retraction medial to malleus/body of incus

M 1 (slight), M 2 (deeper end visible), M 3 (end not visible)

Note for I, II, III and M "U” is used if classification cannot be made due to active disease. atelectasis. For attic retractions the classification is a modification of that of Tos [3] with bone erosion being recorded separately.

The classification also recognises retractions that are progressing beyond atelectasis where the drum starts to retract medial to the malleus or body of the incus assigning these an $\mathrm{M}$ (1 slight, 2 deeper but the end is visible, and 3 deeper with the end not being visible, similar to that used for the attic area in Table 1(b)).

The classification allows recording of activity with a letter designating the type and for keratin $\mathrm{R}$ means fully removable and $\mathrm{N}$ not fully removable. Numbers were used intentionally for the Pars Tensa subgroups so that they can be combined to give an overall score of atelectasis. Although there are various possible methods for calculating a score, in the present study the number in the pars tensa subclassification was taken at its face value giving a score varying between zero for a normal pars tensa and 11 for complete atelectasis. The attic group was graded 1, 2, or 3 and bone erosion of the outer attic wall as yes or no.

After a few weeks of using the classification regularly it is easy to remember, however, it was realised that initially it was difficult to use without familiarisation. In view of this the classification was adapted to an access database (Microsoft Corporation, Figure 2). To incorporate photographs of retractions into the database DbPix (Ammara Digital Image Solutions) was used. This company does offer free trial software but DBpix will be written above the image. Once DPpix is installed in the computer the driver will allow incorporation of photographs into the database by dragging and dropping. The database was programmed to give output reports for the retraction classification (Figure 3) and also the numerical total (Figure 4).

The study involved ten otolaryngologists who were asked to classify seven tympanic membranes with a good variety of multiple retractions (Figure 5) giving a total of 280 observational points. Five were asked to reclassify the retractions after 3 months.

Intraclass correalation and Cronbach's alpha were calculated for pars tensa, attic, and bone erosion. For retest Wilcoxon's signed rank sum test was used for pars tensa and attic, and McNemar test for bone erosion, using Statistics Program for the Social Sciences (SPSS) version 10.

\section{Results}

The results indicate that the classification with the database has good reliability for test and retest, validated by 10 otolaryngologists.

Intraclass correlation was extremely high in all of the groups, pars tensa, attic, and bone erosion (Table 2). The 


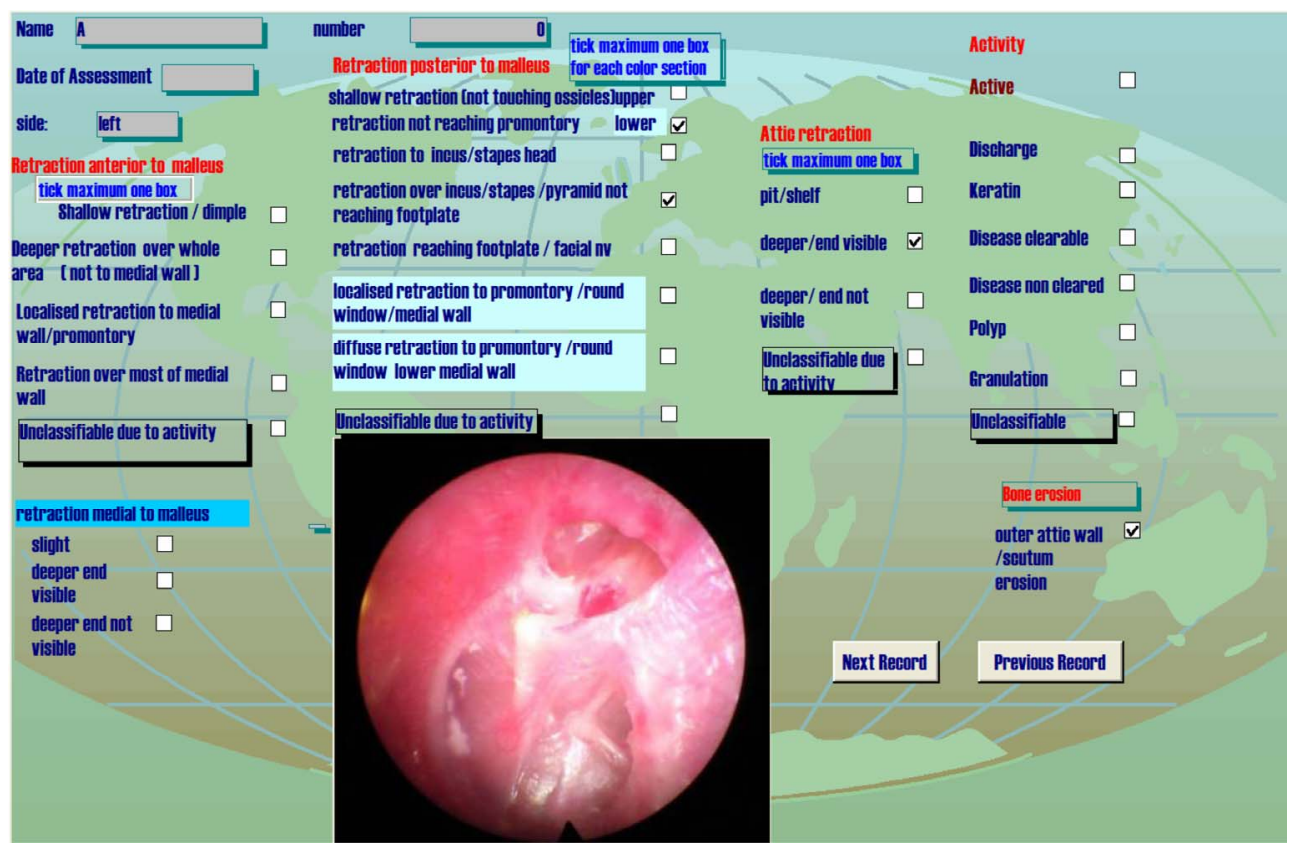

Figure 2. Screen shot of input field of the database, classifying a retraction.

\section{CLASSIFICATION}

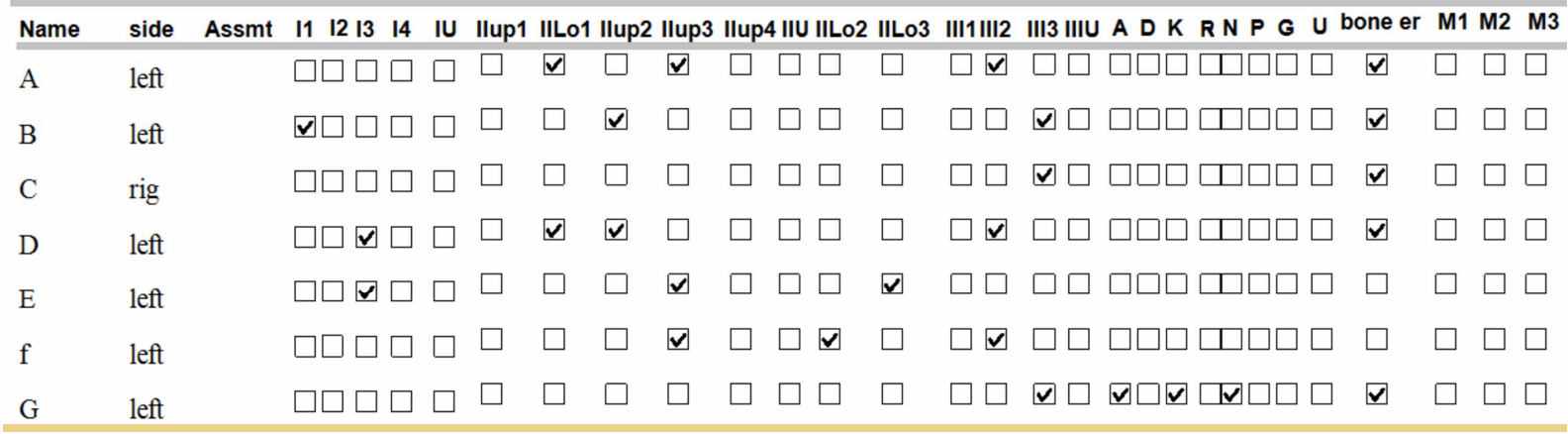

Figure 3. Output field from the database showing classification of the retractions used, labelled A to G in the study.

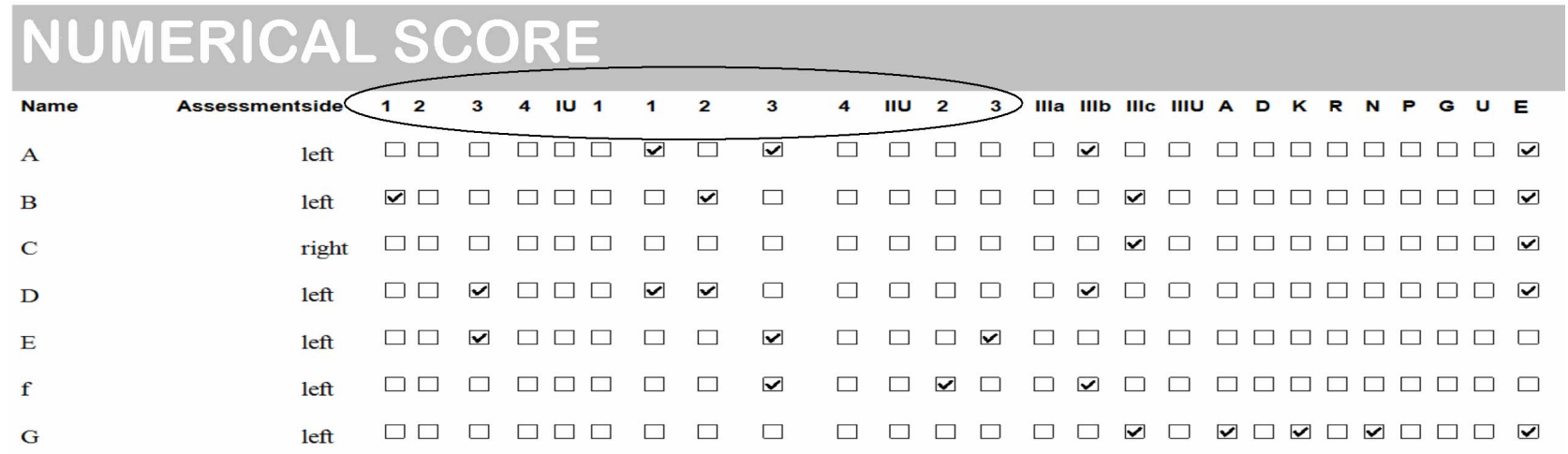

Figure 4. Output field from the database showing the numerical value of retraction for each subgroup of the pars tensa.

Cronbach's alpha was also significantly high in all of the groups, indicating that the scale used has good consis- tency and contributes to validity of the whole scale used (Table 3). 


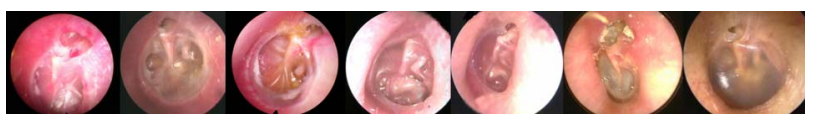

Figure 5. The retraction photographs used in the study.

Table 2. Results of intra-class correalation between observers.

\begin{tabular}{|c|c|c|}
\hline & & 95\% confidence interval \\
\hline - $\quad$ Pars tensa & 0.97 & $0.91-0.99$ \\
\hline - Attic & 0.96 & $0.80-0.99$ \\
\hline - Bone erosion & 0.92 & $0.79-0.98$ \\
\hline
\end{tabular}

Table 3. Results of Chronbach's alpha.

\begin{tabular}{ll}
\hline - Pars tensa & 0.98 \\
- Attic & 0.92 \\
- Bone erosion & 0.96 \\
\hline
\end{tabular}

For retest no significant difference was noted between the first and second test. Although the p value for the attic classification came close to significance with value of 0.057 , the $95 \%$ confidence interval for the difference in medians is 0.5 or less, which being less than 1 shows that this is not significant (Table 4).

\section{Discussion}

Since the original Sade classification there have been many subsequent classifications of tympanic membrane retaction. For the most part these are modifications of the Sade classification and usually involve broad subgroups.

Ohnishi used 2 classess for active and inactive ears with 5 stages of retraction from mild, to severe, ossicular involvement, cholesteatoma, and inner ear involvement [4]. This, however, does not reflect the typical progression of disease in clinical practise. For instance it is possible to have a large attic/antral cholesteatoma without ossicular involvement or equally a small cholesteatoma with ossicular erosion, depending on the initial site and direction of progess. Characon used broad groups based on accessability and controllability, with the retraction being mobile, fixed, to undeterminable (end not visible) [5]. Following this Bours adapted use of endoscopy to the classification [6]. The Erasmus classification again modifies that of Sade with the stage for ossicles and promontory reversed and a deeper retraction group is included [7]. However, whether retraction reaches the promontory or ossicles first is not necessarily a stage of disease but simply related to where the retraction starts. Therefore, previous classifications lose distinction between the anatomical site of the retraction and the pathophysiological advancement of a retraction.
Table 4. Results of retesting observers.

\begin{tabular}{lc}
\hline & Wilcoxon \\
\hline - Pars Tensa & p: 0.142 \\
& p: 0.057 \\
- Bone Erosion & McNemar \\
\hline
\end{tabular}

As demonstrated in the study photographs (Figure 5) not uncommonly attic and pars tensa retractions coexist and it is desirable to have a classification that encompasses the whole drum. The classification will classify the vast majority of retractions but has not been extended outwith the area other than including the presence or absence of erosion of the outer attic wall and there is the possibility adapt the classification to quantify this. Assessment of the ossicles is not included as there is no way of predicting ossiclar erosion with the progress of any individual retraction. To include this would be much more complex and therefore existing ossicular classifications should be used separately. This, can lead to some subjectivity in the II upp group when the long process of the incus and stapes superstructure are missing, making it necessary to estimate the severity between 2 , or 3 on the scale. Similarly audiometry should also be recorded separately.

It would be possible to divide the II low 1 category for the posterior inferior drum into 1(a) and 1(b), which in turn would give a total score out of 12 rather than 11 . However often at this area the next stage on from a slight retraction is to contact the promontory and without other landmarks this might cause more subjectivity in assessment. Therefore, in the study the simpler score for II low out of 3 was used. It is acknowledged that it is possible to have a deeper more posterior retraction not touching the promontory which would still be classified as II low 1 . However, functionally this difference is likely to be less significant.

Central retractions below the umbo, are classified by the predominant part being, either anterior or posterior as long as the involvement of the opposite segment i.e I or II is minimal. However, if both the anterior and posterior drum are involved other than minimally they should be classified separately. A small central retraction below the malleus would be classed as anterior.

Reliability has been demonstrated for inter and intra observer variability which has not been shown for any classification previously. The Cronbach's alpha also implies that the range of retraction in the photographs is good. This classification can detect small changes in retraction which will be useful in follow up or in assessing results and backwards compatability with Sade is possible but adhesion would need to be recorded. The overall score of degree of retraction of the Pars Tensa, zero to 11 
can give a rough assessment of the percentage of atelectasis by multiplying the score by 9 .

The database is easy to use especially when combined with photographic insertion, and it has been shown that photographic documentation of retractions is more accurate than drawings [8]. Photographs, however still need to be classified and with the use of this database there is a reliable method of classification. This lends itself to use by nurse practitioners or allied health professionals who can photograph the retraction enter it into the database, carry out the classification and arrange audiometry. This is likely to be more effective for monitoring retractions over longer periods as only 10 to 20 per cent will progress [1]. Any changes detected can then be notified to the surgeon.

\section{Conclusion}

This classification shows reliabiliy and validity. It allows detection of small changes in tympanic membrane retraction especially affecting the pars tensa but allows classification of the whole tympanic membrane. Although it may seem complicated at first sight it is easy to use with a database.

\section{REFERENCES}

[1] J. Sade, S. Avraham and M. Brown, "Atelectasis, Retraction Pockets and Cholesteatoma," Acta Oto-Laryngologica, Vol. 92, No. 1-6, 1981, pp. 501-512. doi:10.3109/00016488109133289

[2] D. Pothier, "Sade and Tos Classifications of the Tympanic Membrane: Ot Reliable?” Clinical Otolaryngology, Vol. 31, No. 3, 2006, p. 249. doi:10.1111/j.1749-4486.2006.01236_15.x

[3] M. Tos, “Can Cholesteatoma Be Prevented?” Cholesteatoma and Mastoid Surgery: Proceedings of 2nd International Conference on Cholesteatoma and Mastoid Surgery, Tel Aviv, 22-27 March 1981, pp. 591-597.

[4] T. Ohnishi, Y. Shirahata, M. Fukami and S. Hongo, “The Atelectatic Ear and Its Classification,” Auris Nasus Larynx, Vol. 12, Suppl. 1, 1985, pp. S211-S213.

[5] R. Charachon, M. Barthez and J. M. Lejeune, "Spontaneous Retraction Pockets in Chronic Otitis Media Medical and Surgical Therapy,” ENT Journal, Vol. 71, No. 11, 1992, pp. 578-583.

[6] A. F. Bours, M. Decat and M. Gersdorff, "Our Classification of Tympanic Membrane Retraction Pockets," Acta Oto-Rhino-Laryngologica, Vol. 52, No. 1, 1998, pp. 2528.

[7] J. Borgstein, T. V. Gerritsma, M. H. Wieringa and I. A. Bruce, "The Erasmus Atelectasis Classification: Proposal of a New Classification for Atelectasis of the Middle Ear in Children," Laryngoscope, Vol. 117, No. 7, 2007, pp. 1255-1259. doi:10.1097/MLG.0b013e31805d0160

[8] D. Pothier and Z. Awad, "Can We Accurately Interpret Drawings of Ears with Retractions?” Clinical Otolaryngology, Vol. 32, No. 1, 2007, pp. 42-59. doi:10.1111/j.1365-2273.2007.01321.x 